\title{
Solvable nonlinear discrete-time evolutions and Diophantine
} findings

Francesco Calogero

To cite this article: Francesco Calogero (2018) Solvable nonlinear discrete-time evolutions and Diophantine findings, Journal of Nonlinear Mathematical Physics 25:4, 515-517, DOI: https://doi.org/10.1080/14029251.2018.1503395

To link to this article: https://doi.org/10.1080/14029251.2018.1503395

Published online: 04 January 2021 
LETTER TO THE EDITOR

\title{
Solvable nonlinear discrete-time evolutions and Diophantine findings
}

\author{
Francesco Calogero \\ Physics Department, University of Rome "La Sapienza", Rome, Italy \\ Istituto Nazionale di Fisica Nucleare, Sezione di Roma 1 \\ francesco.calogero@uniromal.it,francesco.calogero@romal.infn.it
}

Received 18 March 2018

Accepted 18 May 2018

\begin{abstract}
Certain nonlinearly-coupled systems of $N$ discrete-time evolution equations are identified, which can be solved by algebraic operations; and some remarkable Diophantine findings are thereby obtained. These results might be useful to test the accuracy of numerical routines yielding the $N$ roots of polynomials of arbitrary degree $N$.
\end{abstract}

\section{Introduction and notation}

As the reader will easily see, the results of this paper amount to a transfer-from continuous to discrete time, via the approach introduced in [1]—of the findings reported in [2] and [3] (see also Chapters 3 and 7 of [4]).

Throughout this paper the following notation is used: $N$ and $L$ are two arbitrary positive integers $(N \geq 2, L \geq 2)$, the indices $n$ and $m$ run from 1 to $N$, the discrete-time variable $\ell=0,1,2, \ldots$ takes all nonnegative integer values, the $N$ dependent variables $z_{n}(\ell)$ are generally complex numbers and, being generally defined (see below) as the $N$ zeros of a polynomial of degree $N$ in its (complex) argument $z$, they are the elements of an unordered set of $N$ elements identified hereafter with the notation $\tilde{z}(\ell)$; likewise in the following the notation $\tilde{f}$ denotes the unordered set of $N$ elements $f_{n}$.

\section{Results}

Proposition 2.1. Consider the system of $N$ second-order discrete-time evolution equations

$$
2 \prod_{m=1}^{N}\left[z_{n}(\ell+2)-z_{m}(\ell+1)\right]-\prod_{m=1}^{N}\left[z_{n}(\ell+2)-z_{m}(\ell)\right]=0 ;
$$

note that this formula provides the unordered set $\tilde{z}(\ell+2)$, the elements of which are the $N$ values $z_{n}(\ell+2)$, as the $N$ zeros of the polynomial of degree $N$ in $z$ defined in terms of the two unordered sets $\tilde{z}(\ell)$ and $\tilde{z}(\ell+1)$ as follows:

$$
P_{N}(z ; \tilde{z}(\ell), \tilde{z}(\ell+1))=2 \prod_{m=1}^{N}\left[z-z_{m}(\ell+1)\right]-\prod_{m=1}^{N}\left[z-z_{m}(\ell)\right] .
$$

Let this system of second-order discrete-time evolution equations, (2.1a), be complemented by the following assignments of the two unordered sets $\tilde{z}(0)$ respectively $\tilde{z}(1)$ of $2 N$ initial data $z_{n}(0)$ 
respectively $z_{n}(1)$ : (i) the $N$ data $z_{n}(0)$ are assigned arbitrarily; (ii) the $N$ data $z_{n}(1)$ are defined-in terms of the parameter $L,(L \neq 1)$, the unordered set $\tilde{z}(0)$, and the unordered set $\tilde{f}$ the elements of which are $N$ arbitrarily assigned (generally complex) numbers $f_{m}$-by the $N$ algebraic equations

$$
\prod_{m=1}^{N}\left[z_{n}(1)-z_{m}(0)\right]+\frac{(-1)^{N}}{L-1} \prod_{m=1}^{N}\left[z_{n}(1)-f_{m}\right]=0 ;
$$

hence these $N$ data $z_{n}(1)$ are the $N$ roots of the polynomial $p_{N}^{(1)}(z ; \tilde{z}(0), \tilde{f} ; L)$, of degree $N$ in $z$, defined as follows in terms of the two unordered sets $\tilde{z}(0)$ and $\tilde{f}$ :

$$
p_{N}^{(1)}(z ; \tilde{z}(0), \tilde{f} ; L)=\prod_{m=1}^{N}\left[z-z_{m}(0)\right]+\frac{(-1)^{N}}{L-1} \prod_{m=1}^{N}\left[z-f_{m}\right] .
$$

The solution $z(\ell)$ of the system of second-order discrete-time evolution equations (2.1a) is then given by the $N$ roots of the following polynomial of degree $N$ in $z$ :

$$
\psi_{N}(z ; \tilde{z}(0), \tilde{f} ; L ; \ell)=\left(\frac{L-\ell}{L}\right) \prod_{m=1}^{N}\left[z-z_{m}(0)\right]+\left(\frac{\ell}{L}\right)(-1)^{N} \prod_{m=1}^{N}\left(z-f_{m}\right) .
$$

Proposition 2.1 is proven in the following Section. In the meantime the reader may immediately verify the validity of the formula (2.3) at $\ell=0$ and-via (2.2b)—at $\ell=1$.

Remark 2.1. Note that-while in the formulation of this Proposition 2.1 we considered the system of $N$ equations (2.2) as determining the $N$ elements of the unordered set $\tilde{z}(1)$ in terms of the $2 N$ elements of the two, arbitrarily assigned, unordered sets $\tilde{z}(0)$ and $\tilde{f}$, this system (2.2) of $N$ algebraic equations might as well be considered to define the $N$ elements $f_{m}$ of the unordered set $\tilde{f}$ in terms of the $2 N$ elements of the two-both then arbitrarily assigned—unordered sets $\tilde{z}(0)$ and $\tilde{z}(1)$.

Corollary 2.1. At $\ell=L$ the unordered set $\tilde{z}(L)$ coincides with the unordered set $\tilde{f}$ :

$$
\tilde{z}(L) \equiv \tilde{f} .
$$

The validity of this Corollary 2.1 is an immediate consequence of the Proposition 2.1, being obtained by setting $\ell=L$ in (2.3). And it has an obvious Diophantine implication if the $N$, a priori arbitrary, numbers $f_{m}$ are chosen to be integers or rationals.

\section{Proof}

The starting point of the proof of Proposition 2.1 is the definition (2.3) of the polynomial $\psi_{N}(z ; \ell)$. The consistency of this definition with the assignment of the initial data $\tilde{z}(0)$ and $\tilde{z}(1)$ has already been noted above. What remains to be proven is that the formula

$$
\psi_{N}(z ; \ell)=\prod_{n=1}^{N}\left[z-z_{n}(\ell)\right]
$$

—which, with $\psi_{N}(z ; \ell)$ defined by (2.3), clearly coincides with the statement of Proposition 2.1implies that the $N$ zeros $z_{n}(\ell)$ satisfy the evolution equation (2.1a). To this end we note that since by 
definition (see (2.3)) the dependence of $\psi_{N}(z ; \ell)$ on the discrete-time variable $\ell$ is linear, $\psi_{N}(z ; \ell)$ satisfies identically the linear second-order difference equation

$$
\psi_{N}(z ; \ell+2)-2 \psi_{N}(z ; \ell+1)+\psi_{N}(z ; \ell)=0 .
$$

For $z=z_{n}(\ell+2)$, via (3.1), this formula implies (2.1a).

Q. E. D.

\section{Envoy}

The result reported in the above Proposition 2.1 is likely to look, at least at first sight, somewhat remarkable, especially in view of the arbitrariness of the assignment of the $2 N$ numbers $z_{n}(0)$ and $f_{n}$ (or, equivalently, $z_{n}(0)$ and $z_{n}(1)$; see the above Remark 2.1). But of course, after its validity has been proven, it shall be considered obvious - as all valid mathematical results in some sense are. A potential application of this finding is as a tool to test the accuracy of numerical routines to compute the zeros of polynomials of arbitrary degree $N$ : by comparing, with the simple explicit outcome detailed in the above Corollary 2.1, the results yielded by the application of such routines in order to solve numerically - from the initial data detailed in Proposition 2.1, up to $\ell=L$ - the discrete-time evolution (2.1); which indeed requires finding the zeros of appropriate polynomials of degree $N$ at every step of this discrete-time evolution. In this context the flexibility implied by the possibility to assign arbitrarily the two integers $N$ and $L$ and the $2 N$, generally complex, numbers $z_{n}(0)$ and $f_{n}$ might be quite useful. Specialists in numerical analysis might be interested to explore in detail the vistas implied by such possibilities: note for instance that, for $N=20$ and $f_{m}=m$, Corollary 2.1 -for any arbitrary assignment of the parameters $L$ and $x_{n}(0)$-yields the 20 zeros of the perfidious Wilkinson polynomial [5].

An extension of the findings reported in this paper to the case in which the finite positive integer $N$ is replaced by $\infty$ is of course possible, see [2].

A (perhaps less elegant) variant of the approach described in this paper-characterized by the replacement of the system of second-order discrete-time evolution equations (2.1a) by systems of first-order discrete-time evolution equations-is of course possible, in analogy to the treatments of the continuous-time cases, see [1], [2] and Chapter 3 of [4].

\section{References}

[1] O. Bihun and F. Calogero, "Generations of solvable discrete-time dynamical systems", J. Math. Phys. 58, 052701 (21 pages) (2017); DOI: 10.1063/1.4982959.

[2] F. Calogero, "Finite and infinite systems of nonlinearly coupled ordinary differential equations the solutions of which feature remarkable Diophantine findings", J. Nonlinear Math. Phys. 25 (3) (2018) 433441.

[3] F. Calogero, "Novel differential algorithm to evaluate all the zeros of any generic polynomial", J. Nonlinear Math. Phys. 24, 469-472 (2017). DOI: 10.1080/14029251.2017.1375685.

[4] F. Calogero, Zeros of Polynomials and Solvable Nonlinear Evolution Equations, Cambridge University Press, Cambridge, U.K., 2018 (in press).

[5] J.H. Wilkinson, "The perfidious polynomial", in Studies in Numerical Analysis, vol. 24, pp. 1-28, 1984; G.H. Golub (editor), Mathematical Association of America, Washington DC, USA. 